\title{
DeepACSA: \\ Automatic segmentation of anatomical cross-sectional area in ultrasound images of human lower limb muscles using deep learning
}

Paul Ritsche ${ }^{1}$ BSc, Philipp Wirth ${ }^{2}$ MSc, Neil J. Cronin ${ }^{3}$ PhD, Fabio Sarto 4 MSc, Marco V. Narici 4 $\mathrm{PhD}$, Oliver Faude ${ }^{{ }^{*}} \mathrm{PhD}$, and Martino V. Franchi $4^{*} \mathrm{PhD}$

${ }^{1}$ Department of Sport, Exercise \& Health, University of Basel, Switzerland

2Lightly AG, Zurich, Switzerland

${ }^{3}$ Neuromuscular Research Centre, Faculty of Sport and Health Sciences, University of Jyvaskyla, Finland

4Department of Biomedical Sciences, University of Padova, Italy

*These authors share last authorship

Corresponding author:

Paul Ritsche

Paul.ritsche@unibas.ch

$+491756647158$

Department of Sport, Exercise \& Health

University of Basel

Grosse Allee 6

4052 Basel 
medRxiv preprint doi: https://doi.org/10.1101/2021.12.27.21268258; this version posted December 30, 2021. The copyright holder for this

preprint (which was not certified by peer review) is the author/funder, who has granted medRxiv a license to display the preprint in

It is made available under a CC-BY-NC 4.0 International license .

Background: Muscle anatomical cross-sectional area (ACSA) is an important parameter that characterizes muscle function and helps to classify the severity of several muscular disorders. Ultrasound is a patient friendly, fast and cheap method of assessing muscle ACSA, but manual analysis of the images is laborious, subjective and requires thorough experience. To date, no open access and fully automated program to segment ACSA in ultrasound images is available. On this basis, we present DeepACSA, a deep learning approach to automatically segment ACSA in panoramic ultrasound images of the human rectus femoris (RF), vastus lateralis (VL), gastrocnemius medialis (GM) and lateralis (GL) muscles.

Methods: We trained convolutional neural networks using 1772 ultrasound images from 153 participants ( 25 females, 128 males; mean age $=38.2$ years, range: $13-78$ ) captured by three experienced operators using three distinct devices. We trained three muscle-specific models to detect ACSA.

Findings: Comparing DeepACSA analysis of the RF to manual analysis resulted in intra-class correlation (ICC) of 0.96 (95\% CI 0.94,0.97), mean difference of $0.31 \mathrm{~cm}^{2}(0.04,0.58)$ and standard error of the differences (SEM) of $0.91 \mathrm{~cm}^{2}(0.47,1.36)$. For the VL, ICC was 0.94 (0.91,0.96), mean difference was $0.25 \mathrm{~cm}^{2}(-0.21,0.7)$ and SEM was $1.55 \mathrm{~cm}^{2}(1.13,1.96)$. The GM/GL muscles demonstrated an ICC of $0.97(0.95,0.98)$, a mean difference of $0.01 \mathrm{~cm}^{2}(-$ $0.25,0.24)$ and a SEM of $0.69 \mathrm{~cm}^{2}(0.52,0.83)$.

Interpretation: DeepACSA provides fast and objective segmentation of lower limb panoramic ultrasound images comparable to manual segmentation and is easy to implement both in research and clinical settings. Inaccurate model predictions occurred predominantly on low-quality images, highlighting the importance of high image quality for accurate prediction.

Keywords-deep learning, image segmentation, muscle, ultrasound, U-net 
medRxiv preprint doi: https://doi.org/10.1101/2021.12.27.21268258; this version posted December 30, 2021. The copyright holder for this preprint (which was not certified by peer review) is the author/funder, who has granted medRxiv a license to display the preprint in

It is made available under a CC-BY-NC 4.0 International license.

\section{RESEARCH IN CONTEXT}

\section{Evidence before this study}

Lower limb muscle cross-sectional area is an important predictor of physical performance, frailty, and it can be used in the diagnosis of sarcopenia or in the monitoring of several muscular disorders. Panoramic ultrasound has been proven valid in obtaining images of human muscles compared to magnetic resonance imaging. Further, ultrasound can be performed on bedside and in patients unable to undergo Magnetic Resonance Imaging, in example intensive care unit patients. However, post-scanning manual segmentation of muscle cross-sectional area is laborious and subjective. Thus, automatization of the segmentation process would benefit both researchers and clinicians. We searched Pubmed from database inception to August 31, 2021, using the search terms "deep learning" OR "machine learning" AND "ultrasound" AND "muscle" AND "cross sectional area". The search yielded 15 results, with two investigations comparing deep learning based analysis of lower limb muscle crosssectional area ultrasound images to manual evaluation. By using the bibliographies of the retrieved articles, we identified another investigation. However, none of the found investigations included panoramic ultrasound images displaying a whole muscle crosssectional area in their data sets.

\section{Added value of this study}

We developed DeepACSA, an open-source tool to automatically segment the anatomical crosssectional area in ultrasound images of human lower limb muscles. This is, to our knowledge, the first deep learning based algorithm segmenting panoramic ultrasound images. In contrast to previously proposed algorithms, we used panoramic ultrasound images. DeepACSA analysis was comparable to manual segmentation and reduced time of analysis. Thus, the value added by this investigation lies in increased efficiency and reduced subjectivity of muscle crosssectional area segmentation. DeepACSA includes a graphical user interface allowing for straight forward implementation.

\section{Implications of all the available evidence}

Incorporating deep learning based algorithms which automate the segmentation of muscle cross-sectional area in clinical practice can reduce analysis effort and operator bias. DeepACSA can be easily implemented in clinical and research settings, allowing for fast evaluation of large image datasets. Research is ongoing to assess the generalizability of our results in ageing and pathological settings, and to other imaging modalities.

\section{Introduction}

The anatomical cross-sectional area of a muscle (ACSA) represents a two-dimensional index of muscle size acquired in the transversal plane. ${ }^{1}$ Muscle ACSA is important for clinical and scientific practice; it is related to the capacity of a muscle to generate force and, as a consequence, to locomotor performance..$^{2,3}$ Recent investigations have shown that muscle ACSA represents a useful parameter in the diagnosis and classification of several muscular disorders as well as the potential monitoring of disease progression..$^{-7}$ Lower limb muscle ACSA is often assessed in the diagnosis of sarcopenia and is strongly associated with frailty..$^{-}$ ${ }^{6}$ Moreover, measuring lower limb muscle mass or ACSA is necessary to assess the extent of induced muscle loss in cachectic, dystrophic, and even intensive care unit patients. ${ }^{7-9}$ Muscle mass is also a predictor of hospital stay duration in patients with moderate to severe COVID19. ${ }^{10}$ Thus, whole muscle ACSA represents a crucial variable when monitoring muscle decline in pathological settings, in response to disuse and ageing, and when investigating muscle adaptations to training in rehabilitation and return to sport scenarios.

Muscle ACSA can be assessed using several techniques such as Magnetic Resonance Imaging (MRI) and computer tomography, or with ultrasound imaging. Due to technical advances, the ability to perform measurements at the bedside, and drastically lower costs compared to other imaging modalities, the use of ultrasound has increased in many research and clinical settings. $4,11,12$ Because of the shape and size of several lower limb muscles, conventional static B-mode ultrasound is often unsuitable to assess whole muscle ACSA. ${ }^{1}$ The main reason for this is the limited field of view of most transducers. ${ }^{1,11}$ Hence, panoramic ultrasound, which is used to obtain a panoramic image of the whole muscle, is employed to circumvent the limitations 
medRxiv preprint doi: https://doi.org/10.1101/2021.12.27.21268258; this version posted December 30, 2021. The copyright holder for this preprint (which was not certified by peer review) is the author/funder, who has granted medRxiv a license to display the preprint in

It is made available under a CC-BY-NC 4.0 International license.

imposed by the field of view of most commercially available transducers ${ }^{1}$. In fact, panoramic ultrasound has previously shown good comparability to MRI and excellent inter-session and inter-rater repeatability.13,14

Once ultrasound is implemented as a routine part of ACSA monitoring, large volumes of image data could be collected, necessitating efficient and reliable analysis methods. To date, ultrasound images of muscle ACSA are mostly evaluated manually. However, manual analysis is subjective, laborious, and requires great experience. ${ }^{15,16}$ Furthermore, manually analysing large image datasets is not only time consuming but also leads to reduced focus and thus error proneness. ${ }^{16}$ Semi-automated and automated algorithms have been developed to accelerate the ACSA segmentation process and reduce the subjectivity of manual segmentation in lower limb muscle ultrasound images. ${ }^{17,18}$ These approaches used sophisticated image processing steps to localize features and objects within an image. However, because of this, their generalisability is limited, and they present inconsistent results depending on image properties.

Making use of deep learning might be advantageous compared to non-trainable image processing alone, especially since convolutional neural networks (CNNs) have shown great promise identifying features in medical ultrasound and MRI scans. ${ }^{12,16}$ U-net structured $\mathrm{CNNs}^{19}$ have inter alia been successfully applied to brain MRI segmentation ${ }^{20}$, carotid plaque segmentation in ultrasound images ${ }^{21}$, as well as lower limb muscle segmentation in ultrasound and MRI images. ${ }^{2-25}$ Previously, Chen et al. ${ }^{24}$ and Marzola et al. ${ }^{22,23}$ presented automatic approaches to segment the ACSA of upper and lower limb muscles, but only in conventional static brightness mode ultrasound images, and not panoramic images.

To our knowledge, the semi-automated ACSAuto program ${ }^{17}$ is the only open-access tool available for analysing panoramic lower limb ultrasound images. Thus, it should be determined whether CNN approaches could be used to fully automate the process of ACSA analysis from panoramic ultrasound images. This could increase objectivity and reduce the time and effort needed for the analysis process. ${ }^{15,22}$ Although manual ACSA segmentation of ultrasound images is the current gold standard, using automatic approaches might increase the evaluation quality.

In this study we present DeepACSA, a python package incorporating U-net based CNNs to automatically segment the ACSA of lower limb muscles in panoramic ultrasound images. DeepACSA is open source and includes a custom graphical user interface (GUI) to allow straightforward use and implementation. The muscles of interest are the $\mathrm{m}$. rectus femoris (RF), m. vastus lateralis (VL), mm. gastrocnemius medialis (GM) and lateralis (GL). Here we describe the DeepACSA package and compare it to manual image analysis and a non-trainable image processing script (ACSAuto) ${ }^{17}$.

\section{METHODS}

\section{Image acquisition and data}

The data used in this study contains images from 143 participants of different age groups (adolescent males: $\mathrm{n}=50,16 \cdot 4$ years (13 to 17), adults: $\mathrm{n}=83$ ( 25 females, 58 males), $26 \cdot 9$ years ( 18 to 40 ), elderly males: $n=10,71 \cdot 5$ years ( 65 to 78$)$ ). We used 602 ultrasound images of the RF, 634 ultrasound images of the VL and 298 ultrasound images of the GM and GL (9 to 36 images per participant) acquired in previous and ongoing studies which received ethical approval from the responsible committees. Images were anonymized and randomly divided into a training set for the models as well as an external validation set, making sure that no image of the same muscle region of one participant appeared in both sets. To increase image variability, we used brightness mode panoramic ultrasonography from three devices (ACUSON Juniper, linear-array $54 \mathrm{~mm}$ probe, 12L3, Acuson 12L3, SIEMENS Healthineers, Erlangen, Germany; Aixplorer Ultimate, linear-array 38mm probe, Superline SL10-2, SuperSonic Imagine, Aix-en-Provence, France; Mylab 70, linear array 47mm probe, Esaote Biomedica, Genova, Italy) collected by three different experienced operators to assess the ACSA of RF, VL, GM and GL. We acquired scans at rest while the participants laid in a supine position with their legs extended and feet on the bed. A guide was mounted to the leg to control the transversal 
medRxiv preprint doi: https://doi.org/10.1101/2021.12.27.21268258; this version posted December 30, 2021. The copyright holder for this preprint (which was not certified by peer review) is the author/funder, who has granted medRxiv a license to display the preprint in

It is made available under a CC-BY-NC 4.0 International license.
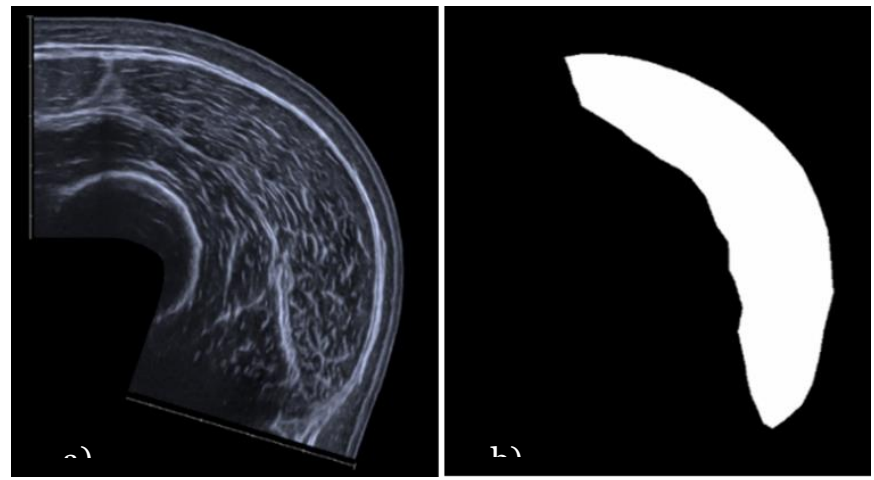

Figure 2: Manual segmentation for convolutional neural network training. a) Original extended-field-ofview ultrasound image of $m$. vastus lateralis, b) manually segmented binary mask of muscle in a).

path. Because of regional differences in muscle size and shape, we acquired images of the RF and VL at $10 \%$ increments from 30 to $70 \%$ of the distance between the lateral femur condyle and the trochanter major. We acquired images of the GM and GL at 30 and 50\% of muscle length. Scans of the RF and VL muscles were either taken separately or cropped from whole quadriceps scans to increase image variability. Ultrasound device settings differed between devices and study protocols, and image depth, brightness and contrast settings were always chosen to ensure best visibility of the muscle. Images included in the training and test sets were manually labelled by an experienced investigator (PR) prior to model training (Fig. 1). Manual segmentation consisted of digitizing the ACSA of each muscle using the polygon tool in FIJI ${ }^{26}$. We randomly included 30 images from the proximal (70\% of femur length), mid (50\% of femur length) and distal (30\% of femur length) regions in the validation set for the RF and VL. For the GM and GL, 37 images from the mid (50\% of muscle length) and 28 images from the proximal (30\% of muscle length) region were randomly included in the validation set. Therefore, the validation set consisted of 90 images for the RF and VL and 65 images for the GM and GL. The ratio of images in the validation and training sets for the RF was $0 \cdot 18$ (90:512), for the VL $0 \cdot 17$ (90:544), and for the GM and GL 0.27 (65:233). No cropped images were included in the validation set because we only had these images available from one device. We measured ACSA in all images of the validation set using the DeepACSA and ACSAuto programs. Because GL segmentation is not supported in ACSAuto, we only analyzed the GM validation images with ACSAuto.
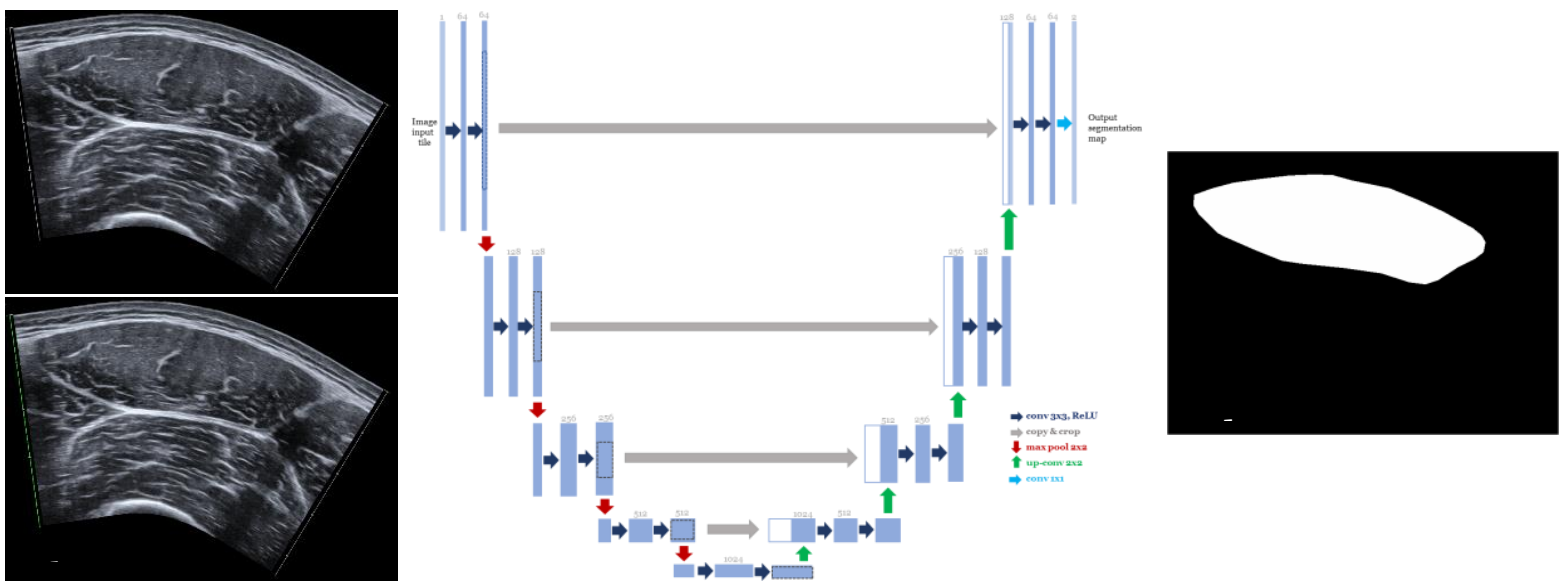

Figure 1: DeepACSA workflow. a) Original ultrasound image of the m. rectus femoris (RF) at 50\% of femur length with automatic scaling (green line). b) The original image is then preprocessed with contrast-limited adaptive histogram equalization and inputted to the model. c) Detailed U-net CNN architecture (modified from Ronneberger et al. ${ }^{21}$ and Cronin et al. ${ }^{15}$ ). Multi-channel feature maps are represented by the blue boxes with number of channels displayed on top of the respective box. Copied feature maps from the convolutional (left) side that are concatenated with the ones from the expanding (right) side are represented by the white boxes. The different operations are marked by the arrows. d) Model prediction of muscle area following post-processing (shown as a binary image). 
medRxiv preprint doi: https://doi.org/10.1101/2021.12.27.21268258; this version posted December 30, 2021. The copyright holder for this preprint (which was not certified by peer review) is the author/funder, who has granted medRxiv a license to display the preprint in

It is made available under a CC-BY-NC 4.0 International license.

\section{Network architecture and training}

We performed sematic segmentation with two classes (ACSA and background) on preprocessed ultrasound images. The image-label pairs (Fig. 1) served as input for the models. The models we employed have a U-net architecture (Fig. 2), consisting of a contracting and an expanding path..$^{15,19}$ The exact architecture of the U-net model can be found elsewhere. ${ }^{15,19}$ The encoder (left) path uses convolution and max pooling layers to generate a 1024-dimensional abstract representation of the input image. Subsequently, the decoder (right) path uses deconvolutional layers to generate a pixelwise class predicton from the abstract image representaion of the input. Features between layers of the same resolution are shared (Fig. 2) so that information about small-scale objects is not lost (hence the U-shape). The model outputs a pixelwise binary label, thus, every pixel of an image is predicted to belong to one of two possible classes. We adopted U-net models due to their demonstrated success for image segmentation in several fields ${ }^{15,22-25}$ and their ability to work well with small datasets containing a few hundred images ${ }^{19}$. Prior to model training, images and masks in the training sets of all muscles were augmented using height and width shift, rotation and horizontal flipping (see shared code for details). We imported, normalized to a scale between 0 and 1 , and resized the images to $256 \times 256$ pixels for training. Training the CNNs with $512 \times 512$-pixel sized images yielded inferior results and was computationally more expensive. Images were preprocessed with contrast-limited adaptive histogram equalization to increase contrast between aponeurosis and muscle tissue. By convention, we applied a random 90/10 \% training/test data split within the training data set. ${ }^{15}$ We trained three separate models, one for $\mathrm{RF}$, one for VL and one for GM and GL combined with the same U-net model architecture. ${ }^{15,19}$ A RTX3060 GPU (NVIDIA, Santa Clara, USA) was used for model training with a defined maximum of 50 epochs and a batch size of one. We used the Adam optimizer for stochastic gradient descent to update network weights with an initial learning rate of $10^{-5} .^{15,19}$ The learning rate was reduced by a factor of 0.1 following ten epochs of loss stagnation. We used binary cross-entropy as a loss function because the segmentation task only represents two classes. To reduce the risk of overfitting, we implemented early stopping when the training error decreased, and the test error reached a plateau or increased. During training, model performance was evaluated using the intersection over union (IoU) measure to test the overlap between manually segmented muscle area and labels predicted by the respective model (Table 1). IoU is defined as

$$
\operatorname{IoU}(A, B)=\frac{|A \cap B|}{|A|+|B|-|A \cap B|},
$$

where $\mathrm{O} \leq \mathrm{IoU}(\mathrm{A}, \mathrm{B}) \leq 1$, A describes the ground truth and $\mathrm{B}$ the model predictions. The IoU is maximal if the ground truth $\mathrm{A}$ and model predictions $\mathrm{B}$ are the same and minimal if $\mathrm{A}$ and $\mathrm{B}$ have no overlap.

The output of each CNN is a binary mask image that contains the predicted muscle area. The output is post-processed so that holes within the predicted muscle are filled and, in case of multiple predicted structures, only the largest structure is kept. The DeepACSA code is written in Python using a Keras API to interface with Tensorflow for model training. The code and trained models from this project are openly available at https://github.com/PaulRitsche/DeepACSA.git. For more information about program use we refer the reader to an instructional online video (Link: https://youtu.be/ItgCqVSNcqM).

\section{Analysis metrics}

All statistical analyses were performed in R software ${ }^{27}$ (Base, BlandAltmanLeh, irr, readxl and rstudioapi packages). The analysis script is shared as a supplementary file. We compared DeepACSA and ACSAuto measurements to manual measurements. For this purpose, we calculated consecutive-pairwise intra-class correlations (ICC) and standard error of the differences between methods (SEM) with 95\% compatibility intervals (CI). We used BlandAltman analysis ${ }^{28}$ to test the agreement between two analysis methods and set the limits of agreement to \pm 1.96 standard deviations. We computed the standardized mean bias according to Hopkins ${ }^{29}$, with $0 \cdot 1,0 \cdot 3,0 \cdot 6,1 \cdot 0$ and $2 \cdot 0$ representing small, moderate, large, very large and extremely large errors respectively. 
medRxiv preprint doi: https://doi.org/10.1101/2021.12.27.21268258; this version posted December 30, 2021. The copyright holder for this preprint (which was not certified by peer review) is the author/funder, who has granted medRxiv a license to display the preprint in

It is made available under a CC-BY-NC 4.0 International license.

\section{RESULTS}

The training of the deep neural networks required 23 to 38 epochs, and IoU and loss metrics are shown in Table 1. An example of pre-processing and model prediction following postprocessing is shown in Figure 2. ICCs, SEMs, mean bias, standardized mean bias with 95\% CI as well as percentage values for all muscles comparing DeepACSA and ACSAuto to manual measurements are shown in Table 2 and Fig. 3. We additionally report comparability statistics after removing incorrect predictions (RF: $5 \cdot 5 \%$, VL: $13 \cdot 3 \%$, GM \& GL: $3 \cdot 1 \%$ ) by the models (Table 2). Predictions were removed following visual inspections by an experienced investigator (PR) based on obvious deviations from the expected ACSA of the respective muscle (Fig. 4). Comparing DeepACSA analysis of all muscles including the wrong predictions to manual analysis resulted in ICCs between 0.94 and 0.99 , mean differences of -0.01 to $0.37 \mathrm{~cm}^{2}$ and SEMs ranging from $0 \cdot 34$ to $1.55 \mathrm{~cm}^{2}$ (Table 2). Calculated standardized mean biases were small for all muscles ranging from -0.02 to $0 \cdot 07$ (Table 2). The comparison of ACSAuto with manual analysis of all images and muscles resulted in ICCs between 0.97 and 0.99, mean differences of -0.13 to $0.24 \mathrm{~cm}^{2}$ and SEMs ranging from 0.23 to $0.66 \mathrm{~cm}^{2}$. Standardized mean biases were small for all muscles ranging from -0.02 to 0.05 (Table 2). DeepACSA segmentation resulted in differences between muscle regions for the RF. At $30 \%$ of femur

Table 1

Final intersection over union and loss functions for the three selected models.

\begin{tabular}{ccccccc}
\hline \hline Metric & \multicolumn{2}{c}{ RF } & \multicolumn{2}{c}{ VL } & \multicolumn{2}{c}{ GM \& GL } \\
\hline & Training & Test & Training & Test & Training & Test \\
\hline IoU & 0.997 & 0.996 & 0.994 & 0.992 & 0.997 & 0.996 \\
Accuracy & 0.986 & 0.984 & 0.975 & 0.971 & 0.975 & 0.973
\end{tabular}

Final intersection over union (Iou) and accuracy metrics for the training and test sets. IoU was calculated for the model prediction and the manually segmented ground truth in the training and the randomly generated test set using the training/test split. M. rectus femoris (RF), m. vastus lateralis $(V L), m m$. gastrocnemius medialis (GM), and lateralis (GL).

Table 2

Comparison of manual analysis and ACSAuto and DeepACSA

\begin{tabular}{|c|c|c|c|c|c|c|c|}
\hline Muscle & Mode & $\begin{array}{c}\text { ICC } \\
(95 \% \mathrm{CI})\end{array}$ & $\begin{array}{l}\mathrm{MD} \mathrm{cm}^{2} \\
(95 \% \mathrm{CI})\end{array}$ & MD \% & $\begin{array}{l}\text { SEM cm² } \\
(95 \% \mathrm{CI})\end{array}$ & $\begin{array}{c}\text { SEM } \\
\%\end{array}$ & Stmd \\
\hline RF & $\begin{array}{l}\text { Manual vs. } \\
\text { ACSAuto }\end{array}$ & $\begin{array}{c}0.996 \\
(0.994 ; 0.998)\end{array}$ & $\begin{array}{c}0 \cdot 24 \\
(0 \cdot 17 ; 0 \cdot 31)\end{array}$ & $2 \cdot 83$ & $\begin{array}{c}0 \cdot 23 \\
(0 \cdot 19 ; 0 \cdot 27)\end{array}$ & $5 \cdot 10$ & $0 \cdot 05$ \\
\hline RF & $\begin{array}{l}\text { Manual vs. } \\
\text { DeepACSA }\end{array}$ & $\begin{array}{c}0.96 \\
(0.939 ; 0 \cdot 973)\end{array}$ & $\begin{array}{c}0 \cdot 31 \\
(0 \cdot 04 ; 0 \cdot 58)\end{array}$ & $3 \cdot 32$ & $\begin{array}{c}0 \cdot 91 \\
(0 \cdot 47 ; 1 \cdot 36)\end{array}$ & $9 \cdot 96$ & $0 \cdot 07$ \\
\hline $\mathrm{RF}$ & $\begin{array}{c}\text { Manual vs. } \\
\text { DeepACSA rm }\end{array}$ & $\begin{array}{c}0.994 \\
(0 \cdot 99 ; 0 \cdot 996)\end{array}$ & $\begin{array}{c}0 \cdot 13 \\
(0 \cdot 02 ; 0 \cdot 24)\end{array}$ & $1 \cdot 90$ & $\begin{array}{c}0 \cdot 34 \\
(0 \cdot 21 ; 0 \cdot 46)\end{array}$ & $6 \cdot 28$ & $0 \cdot 03$ \\
\hline VL & $\begin{array}{l}\text { Manual vs. } \\
\text { ACSAuto }\end{array}$ & $\begin{array}{c}0.988 \\
(0.982 ; 0.992)\end{array}$ & $\begin{array}{c}-0 \cdot 13 \\
(-0 \cdot 33 ; 0 \cdot 06)\end{array}$ & $-0 \cdot 89$ & $\begin{array}{c}0 \cdot 66 \\
(0 \cdot 55 ; 0 \cdot 77)\end{array}$ & $3 \cdot 13$ & -0.02 \\
\hline VL & $\begin{array}{l}\text { Manual vs. } \\
\text { DeepACSA }\end{array}$ & $\begin{array}{c}0.941 \\
(0.911 ; 0.96)\end{array}$ & $\begin{array}{c}0 \cdot 25 \\
(-0 \cdot 21 ; 0 \cdot 70)\end{array}$ & $1 \cdot 74$ & $\begin{array}{c}1 \cdot 55 \\
(1 \cdot 13 ; 1 \cdot 96)\end{array}$ & $6 \cdot 80$ & $0 \cdot 04$ \\
\hline VL & $\begin{array}{c}\text { Manual vs. } \\
\text { DeepACSA rm }\end{array}$ & $\begin{array}{c}0.977 \\
(0.963 ; 0.985)\end{array}$ & $\begin{array}{c}0 \cdot 37 \\
(0 \cdot 09 ; 0 \cdot 65)\end{array}$ & $2 \cdot 26$ & $\begin{array}{c}0 \cdot 85 \\
(0 \cdot 71 ; 0 \cdot 97)\end{array}$ & $4 \cdot 74$ & $0 \cdot 06$ \\
\hline GM\&GL & $\begin{array}{c}\text { Manual vs. } \\
\text { ACSAuto }\end{array}$ & $\begin{array}{c}0.97 \\
(0.94 ; 0.985)\end{array}$ & $\begin{array}{c}0 \cdot 06 \\
(-0 \cdot 2 ; 0 \cdot 32)\end{array}$ & $0 \cdot 28$ & $\begin{array}{c}0 \cdot 51 \\
(0 \cdot 35 ; 0 \cdot 67)\end{array}$ & $4 \cdot 19$ & $0 \cdot 02$ \\
\hline GM\&GL & $\begin{array}{l}\text { Manual vs. } \\
\text { DeepACSA }\end{array}$ & $\begin{array}{c}0.968 \\
(0 \cdot 948 ; 0 \cdot 98)\end{array}$ & $\begin{array}{c}-0.01 \\
(-0 \cdot 25 ; 0 \cdot 24)\end{array}$ & $-1 \cdot 47$ & $\begin{array}{c}0.69 \\
(0.52 ; 0 \cdot 83)\end{array}$ & $8 \cdot 14$ & $0 \cdot 00$ \\
\hline GM\&GL & $\begin{array}{c}\text { Manual vs. } \\
\text { DeepACSA rm }\end{array}$ & $\begin{array}{c}0.983 \\
(0.972 ; 0.99)\end{array}$ & $\begin{array}{c}0 \cdot 26 \\
(0 \cdot 09 ; 0 \cdot 44)\end{array}$ & $2 \cdot 08$ & $\begin{array}{c}0 \cdot 46 \\
(0 \cdot 34 ; 0 \cdot 57)\end{array}$ & $4 \cdot 18$ & $0 \cdot 07$ \\
\hline
\end{tabular}

$R F=$ rectus femoris, $V L=$ vastus lateralis, $G M \& G L=$ gastrocnemii, $r m=$ wrong prediction removed , $I C C=$ Intra-class correlation, $C I=$ compatibility interval, $M D=$ mean difference, $S E M=$ Standard error of the differences, and Stmd = standardized mean difference. 
medRxiv preprint doi: https://doi.org/10.1101/2021.12.27.21268258; this version posted December 30, 2021. The copyright holder for this preprint (which was not certified by peer review) is the author/funder, who has granted medRxiv a license to display the preprint in perpetuity.

It is made available under a CC-BY-NC 4.0 International license .

length, the ICC was $0 \cdot 503(95 \% \mathrm{CI}: 0 \cdot 18 ; 0 \cdot 73)$, the mean difference was $0 \cdot 79 \mathrm{~cm}^{2}(0 \cdot 03 ; 1 \cdot 55)$ and the SEM was $1.44 \mathrm{~cm}^{2}(0 \cdot 69 ; 2 \cdot 12)$. Standardized mean bias was moderate $(0 \cdot 40)$. In contrast, at $50 \%$ of femur length, the ICC was $0 \cdot 91(0 \cdot 83 ; 0 \cdot 96)$, the mean difference was $0 \cdot 07$ $\mathrm{cm}^{2}(-0 \cdot 21 ; 0 \cdot 36)$ and the SEM was $0.54 \mathrm{~cm}^{2}(0 \cdot 18 ; 0 \cdot 86)$. Standardized mean bias was small (0.04). At 70\% of femur length, the ICC was $0.97(0 \cdot 94 ; 0 \cdot 99)$, the mean difference was 0.06 $\mathrm{cm}^{2}(-0 \cdot 03 ; 0 \cdot 15)$ and the SEM was $0.18 \mathrm{~cm}^{2}(0.11 ; 0.23)$. Similar to the $50 \%$ of femur length site, the observed standardized mean bias was small (0.05). Regional data for VL, GM \& GL can be found in the supplementary material. Removing incorrect model predictions resulted in increased ICCs and decreased mean differences, SEMs, and standardized mean bias for all muscles (Table 2). In Bland-Altman analysis, concerns regarding heteroscedasticity were only raised when comparing manual to DeepACSA RF analysis. With increasing muscle size, it seems that the variance around the mean difference increases.

Additionally, we compared the efficiency of DeepACSA and ACSAuto. Whereas analysis duration was 4.0s standard deviation (SD) \pm 0.43 for analyzing one image in our validation set usind DeepACSA, analysis duration for one image using ACSAuto was 40.3S \pm SD 6.9.
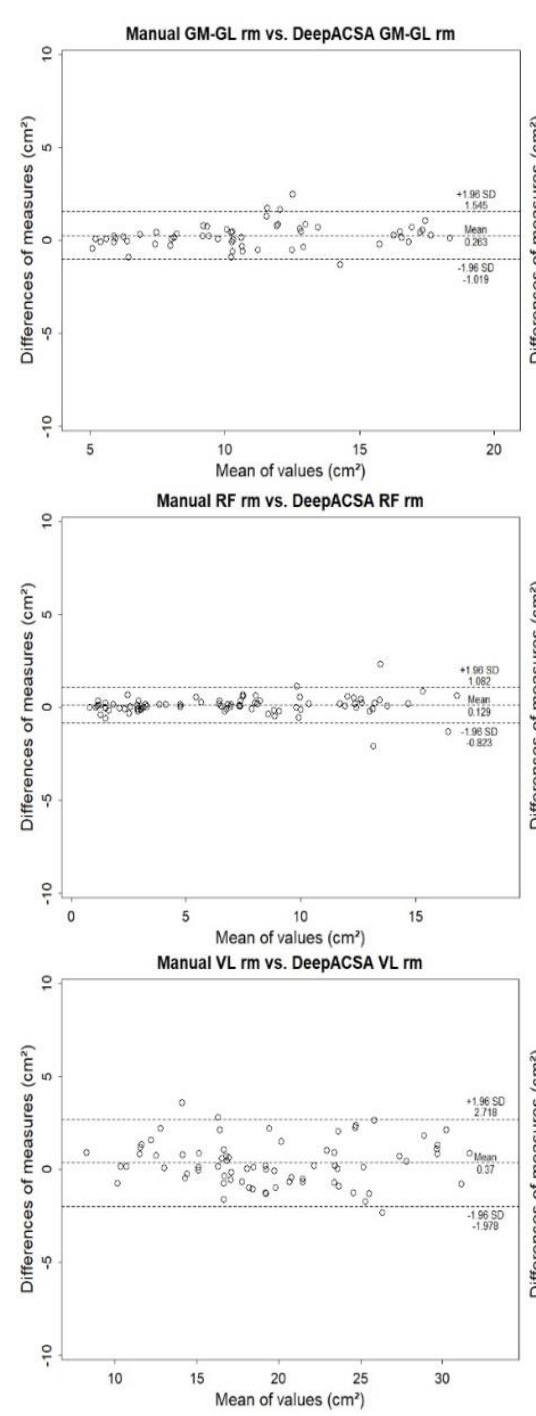

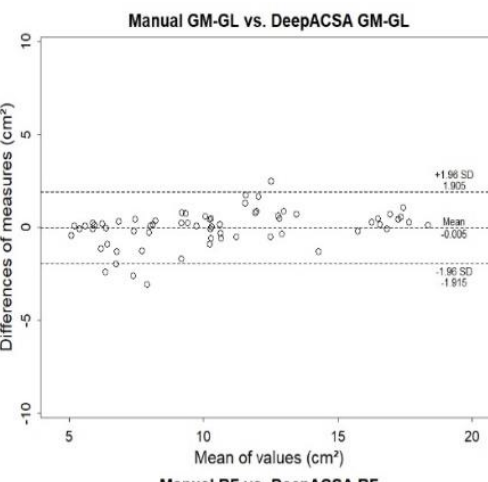

Manual RF vs. DeepACSA RF
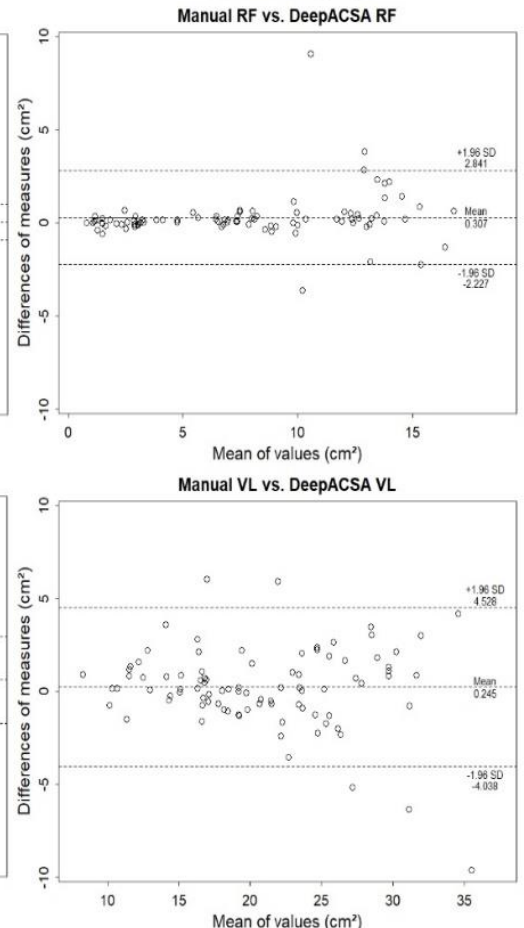
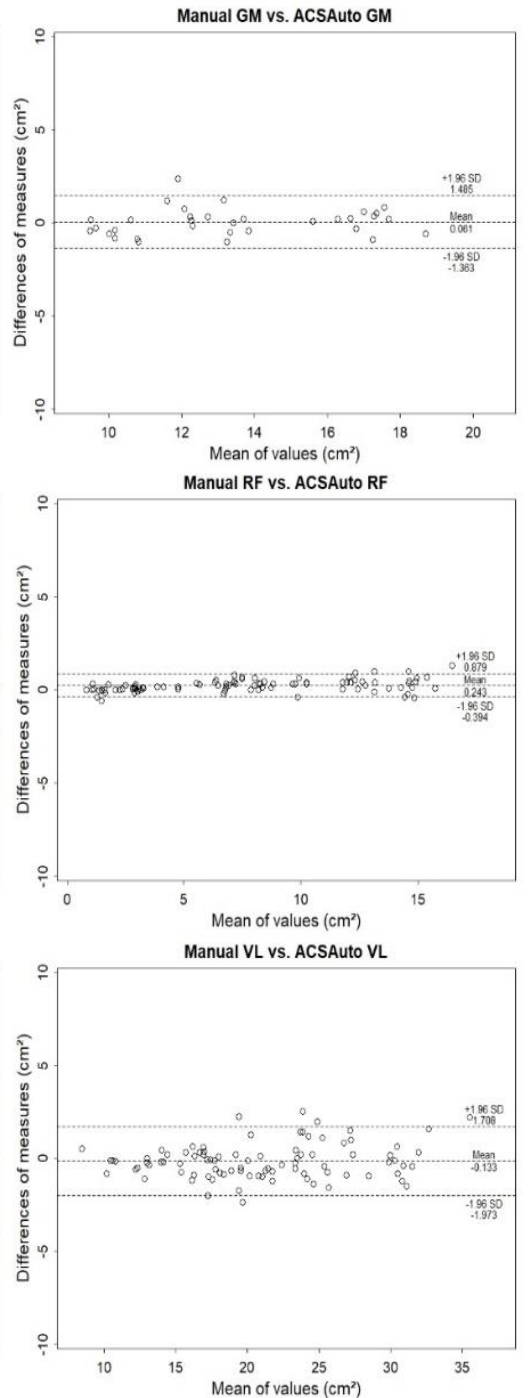

Figure 3: Bland-Altman plots of all muscles plotting the difference between manual and DeepACSA with incorrect predictions removed (rm), manual and DeepACSA as well as manual and ACSAuto area segmentation measurements against the mean of both measures. Dotted and solid lines illustrate 95\% limits of agreement and bias. $M$. rectus femoris $(R F)$ and $m$. vastus lateralis $(V L), m m$. gastrocnemius medialis $(G M)$, and lateralis $(G L)$. 


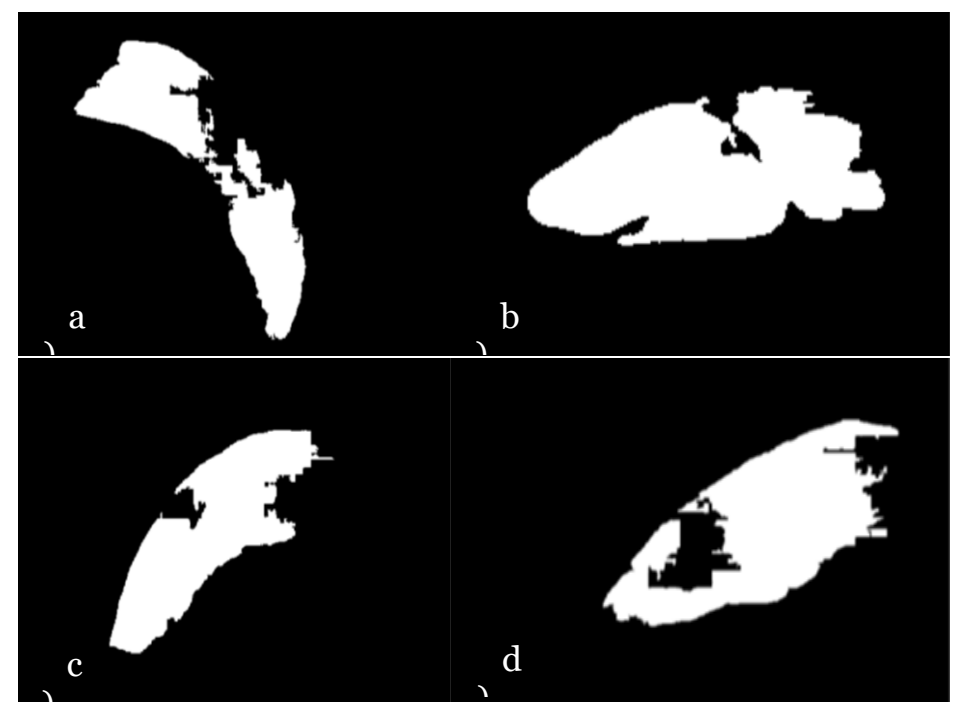

Figure 4: Examples of incorrect predictions and obvious deviations from the expected ACSA of the respective muscles. a) m. vastus lateralis, b) $m$. rectus femoris, c) m. gastrocnemius medialis, and d) m. gastrocnemius lateralis.

\section{Discussion}

Here we present DeepACSA, a deep learning approach for automatic analysis of muscle ACSA in panoramic brightness mode ultrasound images. The predictions of our trained CNNs were comparable to manual segmentation for all the lower limb muscles investigated. To our knowledge, DeepACSA represents the first openly accessible program able to automatically segment the muscle area of rectus femoris, vastus lateralis, gastrocnemius medialis, and gastrocnemius lateralis in panoramic ultrasound images.

Comparing the DeepACSA-based analysis of all muscles, the predictions of GM \& GL ACSA were found to have the highest agreement with manual analysis. The worst agreement was observed for the RF, which was also the only muscle that displayed regional differences. Most incorrect RF predictions occurred at 30\% of femur length. Here the aponeuroses of the RF are more difficult to scan, and thus the image quality decreases. More distally, at $70 \%$ of femur length, the RF is usually small, sometimes non-existent, and the echogenicity is increased, leading to reduced overall pixel contrast. Small muscle area and lower pixel contrast make prediction more difficult and might lead to deletion or selection of the wrong area during postprocessing.

All our CNNs demonstrated minimal loss and high IoU scores. Although high IoU and minimal loss values in the training set might indicate overfitting, similar values were observed for the test set. Thus, our model can generalize to unseen data. However, we used ultrasound images of all three devices in our training, test and validation sets leading to similar image characteristics in all data sets. To test whether our CNNs can generalize to unseen image data from other devices, images from a fourth device should solely be added to the validation set. Based on our results, visual inspection of the model output is still necessary, and the removal of erroneous predictions resulted in increased comparability for all muscles. However, we believe that adding more training data might help to eliminate these model failures. Erroneous predictions in the validation set mostly occurred when image quality was low, i.e. when tissue contrast was low or pixel values were homogeneous. Therefore, image quality is still an important factor for correct muscle ACSA segmentation using deep learning. Images for which muscle ACSA was predicted incorrectly should be analyzed using ASCAuto ${ }^{17}$ or manually.

Given that DeepACSA is comparable to manual evaluation, the program might be implementable in a clinical setting as well. In contrast to MRI or computer tomography, ultrasound can be used quickly at the bedside of patients and practitioners do not necessarily need active cooperation from patients. ${ }^{4}$ This might be especially important for patients in the intensive care unit or with severe muscular disorders.9,30 DeepACSA analysis is comparable to manual evaluation, more time efficient, and automates the segmentation process. This is 
medRxiv preprint doi: https://doi.org/10.1101/2021.12.27.21268258; this version posted December 30, 2021. The copyright holder for this preprint (which was not certified by peer review) is the author/funder, who has granted medRxiv a license to display the preprint in

It is made available under a CC-BY-NC 4.0 International license.

important for clinical practice as it decreases the effort needed to analyze ultrasound image data. In fact, reducing the subjectivity of the analysis might increase the overall validity of clinical results. Moreover, our trained CNNs could be used to correctly predict the ACSA of other muscles, in patients with muscular diseases or an ageing population (for example when screening for the presence of sarcopenia). However, because of differences in image properties, it might be necessary to retrain our models including labeled images from the respective muscles or clinical populations in the training dataset. In the future, we aim to release more models that allow data from additional muscles and clinical populations to be analysed using the DeepACSA package.

In contrast to the algorithms presented by Chen et al. ${ }^{24}$ and Marzola et al. ${ }^{22,23}$, who used a supervised learning approach with U-net structured CNNs and achieved good precision and recall rates, DeepACSA can segment muscles in panoramic ultrasound images. To our knowledge, DeepACSA is the only program that includes images of several lower limb muscles from various muscle regions, different operators, and several ultrasound devices. Whereas Marzola et al. ${ }^{22}$ included images from the tibialis anterior, GM and biceps brachii at rest, Chen et al. ${ }^{24}$ included images of the RF during contraction. Acquiring images during contraction could limit the generalizability of the model to images acquired at rest. Furthermore, while the algorithms proposed by Marzola et al.22,23 seem to distinguish between clinical and healthy populations based on echogenicity values, segmenting ACSA in muscles that exceed the field of view of the ultrasound transducer may have limited meaningfulness.

It is important to note that the predictions of our trained CNNs for all muscles showed lower agreement with manual analysis when compared to ACSAuto (DeepACSA: 95\% limits of agreement 4.5 to $-4 \cdot 0 \mathrm{~cm}^{2}$, ACSAuto: $1 \cdot 7$ to $-1 \cdot 9 \mathrm{~cm}^{2}$ ). This is due to the manual correction of the suggested outline during ACSAuto analysis. Yet, the feature detection filters used for image segmentation in ACSAuto are highly dependent on image properties. Furthermore, ACSAuto is somewhat subjective because the user must validate the proposed muscle outline. In addition, not correcting the suggested outline led to large measurement errors compared to manual analysis and thus unusable results. ${ }^{17}$ By employing trainable CNNs, DeepACSA is more robust to variation in ultrasound image pixel characteristics because the whole image texture is considered, and more complex features are computed. Additionally, DeepACSA reduces user bias in the analysis process, as no user input is required during image segmentation.

This investigation has some limitations. First, we performed no cross-validation or hyper parameter tuning for our models. ${ }^{16}$ Additionally, comparing several different deep neural networks with various architectures could be beneficial to determine the ideal model for this task. However, the U-net architecture we employed demonstrated good results when segmenting muscle aponeuroses and fascicles in sagittal plane ultrasound images of the VL and GM. ${ }^{15}$ Although our training set consisted of data from different age groups, we only included images from adolescent and young healthy participants and three devices in our validation sets because of limited available data from elderly people. Thus, we cannot yet generalize our results to older people, clinical populations or other devices.22,23 Finally, DeepACSA is currently able to automatically evaluate single muscle images but not videos.

\section{CONCLUSION}

DeepACSA segmentation of panoramic ultrasound images from rectus femoris, vastus lateralis, gastrocnemius medialis and lateralis muscles yielded comparable results to manual segmentation. Therefore, our trained convolutional neural networks can automatically segment lower limb muscles in panoramic ultrasound images. DeepACSA objectifies and accelerates the evaluation process of panoramic ACSA ultrasound images, allowing large datasets to be evaluated quickly, and representing a valuable tool that can be implemented in clinical settings. However, our results demonstrated that visual inspection of the output may still be necessary to optimize predictions and avoid misclassifications. In the future, the segmentation performance of different model architectures should be compared whilst using a larger, more variable training dataset. 
medRxiv preprint doi: https://doi.org/10.1101/2021.12.27.21268258; this version posted December 30, 2021. The copyright holder for this preprint (which was not certified by peer review) is the author/funder, who has granted medRxiv a license to display the preprint in

It is made available under a CC-BY-NC 4.0 International license .

\section{DECLARATION OF INTEREST}

All authors report no competing interest.

\section{DATA SHARING STATEMENT}

All data used in this study is publicly available. The DeepACSA package is available on Github at https://github.com/PaulRitsche/DeepACSA.git. Our trained models are available at https://doi.org/10.5281/zenodo.5799068. Anonymized panoramic ultrasound images are available at https://doi.org/10.5281/zenodo.5799204. The instructional video can be accessed using this link https://youtu.be/ItgCqVSNc9M.

\section{CONTRIBUTORS}

P.R., O.F., and M.V.F designed the study. M.V.N. and N.J.C. assisted in designing the study. P.R., P.W., and N.J.C. wrote the DeepACSA package code. P.R., F.S., and M.V.F. collected the ultrasound images. P.R. and M.V.F. performed annotations and quality control. P.R. and P.W. performed the deep learning analysis and visualizations. P.R., O.F. and M.V.F wrote the first draft of the manuscript. All authors read and revised the manuscript draft and approved the final manuscript. All authors had access to all the data in the study and P.R., O.F. and M.V.F verified the data and had final responsibility for the decision to submit for publication.

\section{ROLE OF FUNDING SOURCE}

This investigation was not supported by any funding source. 
medRxiv preprint doi: https://doi.org/10.1101/2021.12.27.21268258; this version posted December 30, 2021. The copyright holder for this preprint (which was not certified by peer review) is the author/funder, who has granted medRxiv a license to display the preprint in

It is made available under a CC-BY-NC 4.0 International license .

\section{REFERENCES}

1 Franchi MV, Raiteri BJ, Longo S, Sinha S, Narici MV, Csapo R. Muscle Architecture Assessment: Strengths, Shortcomings and New Frontiers of in Vivo Imaging Techniques. Ultrasound Med Biol 2018; 44: 2492-504.

2 Evangelidis PE, Massey GJ, Pain MTG, Folland JP. Strength and size relationships of the quadriceps and hamstrings with special reference to reciprocal muscle balance. Eur J Appl Physiol 2016; 116: 593-600.

3 Ritsche P, Bernhard T, Roth R, et al. M. Biceps Femoris Long Head Architecture and Sprint Ability in Youth Soccer Players. Int J Sports Physiol Perform 2020; : 1-9.

4 Ticinesi A, Narici MV, Lauretani F, et al. Assessing sarcopenia with vastus lateralis muscle ultrasound: an operative protocol. Aging Clin Exp Res 2018; 30: 1437-43.

5 Chianca V, Albano D, Messina C, et al. Sarcopenia: imaging assessment and clinical application. Abdom Radiol 2021; published online Oct 23. DOI:10.1007/s00261-02103294-3.

6 Mueller N, Murthy S, Tainter CR, et al. Can Sarcopenia Quantified by Ultrasound of the Rectus Femoris Muscle Predict Adverse Outcome of Surgical Intensive Care Unit Patients as well as Frailty? A Prospective, Observational Cohort Study. Ann Surg 2016; 264: 111624.

7 Anderson LJ, Chong N, Migula D, et al. Muscle mass, not radiodensity, predicts physical function in cancer patients with or without cachexia. Oncotarget 2020; 11: 1911-21.

8 Jacques MF, Onambele-Pearson GL, Reeves ND, Stebbings GK, Smith J, Morse CI. Relationships between muscle size, strength, and physical activity in adults with muscular dystrophy: Muscle strength, size and physical activity in muscular dystrophy. $J$ Cachexia Sarcopenia Muscle 2018; 9: 1042-52.

9 Puthucheary ZA, Rawal J, McPhail M, et al. Acute Skeletal Muscle Wasting in Critical Illness. JAMA 2013; 310: 1591.

10 Gil S, Jacob Filho W, Shinjo SK, et al. Muscle strength and muscle mass as predictors of hospital length of stay in patients with moderate to severe COVID-19: a prospective observational study. J Cachexia Sarcopenia Muscle 2021; : jcsm.12789.

11 Sarto F, Spörri J, Fitze DP, Quinlan JI, Narici MV, Franchi MV. Implementing Ultrasound Imaging for the Assessment of Muscle and Tendon Properties in Elite Sports: Practical Aspects, Methodological Considerations and Future Directions. Sports Med 2021; 51: 115170 .

12 Shin Y, Yang J, Lee YH, Kim S. Artificial intelligence in musculoskeletal ultrasound imaging. Ultrasonography 2021; 40: 30-44.

13 Noorkoiv M, Nosaka K, Blazevich AJ. Assessment of quadriceps muscle cross-sectional area by ultrasound extended-field-of-view imaging. Eur J Appl Physiol 2010; 109: 631-9.

14 Bolsterlee B, Gandevia SC, Herbert RD. Ultrasound imaging of the human medial gastrocnemius muscle: how to orient the transducer so that muscle fascicles lie in the image plane. J Biomech 2016; 49: 1002-8.

15 Cronin NJ, Finni T, Seynnes O. Fully automated analysis of muscle architecture from Bmode ultrasound images with deep learning. ArXiv20090479o Cs Eess 2020; published online Sept 10. http://arxiv.org/abs/2009.04790 (accessed Aug 12, 2021).

16 Greenspan H, van Ginneken B, Summers RM. Guest Editorial Deep Learning in Medical Imaging: Overview and Future Promise of an Exciting New Technique. IEEE Trans Med Imaging 2016; 35: 1153-9.

17 Ritsche P, Wirth P, Franchi MV, Faude O. ACSAuto-semi-automatic assessment of human vastus lateralis and rectus femoris cross-sectional area in ultrasound images. Sci Rep 2021; 11: 13042. 
medRxiv preprint doi: https://doi.org/10.1101/2021.12.27.21268258; this version posted December 30, 2021. The copyright holder for this preprint (which was not certified by peer review) is the author/funder, who has granted medRxiv a license to display the preprint in It is made available under a CC-BY-NC 4.0 International license .

18 Salvi M, Caresio C, Meiburger KM, De Santi B, Molinari F, Minetto MA. Transverse Muscle Ultrasound Analysis (TRAMA): Robust and Accurate Segmentation of Muscle CrossSectional Area. Ultrasound Med Biol 2019; 45: 672-83.

19 Ronneberger O, Fischer P, Brox T. U-Net: Convolutional Networks for Biomedical Image Segmentation. ArXiv150504597 Cs 2015; published online May 18. http://arxiv.org/abs/1505.04597 (accessed Oct 25, 2021).

20Lee B, Yamanakkanavar N, Choi JY. Automatic segmentation of brain MRI using a novel patch-wise U-net deep architecture. PLOS ONE 2020; 15: e0236493.

21 Meshram NH, Mitchell CC, Wilbrand S, Dempsey RJ, Varghese T. Deep Learning for Carotid Plaque Segmentation using a Dilated U-Net Architecture. Ultrason Imaging 2020; 42: 221-30.

22 Marzola F, van Alfen N, Doorduin J, Meiburger KM. Deep learning segmentation of transverse musculoskeletal ultrasound images for neuromuscular disease assessment. Comput Biol Med 2021; 135: 104623.

23 Marzola F, Alfen N van, Salvi M, Santi BD, Doorduin J, Meiburger KM. Automatic segmentation of ultrasound images of gastrocnemius medialis with different echogenicity levels using convolutional neural networks. In: 2020 42nd Annual International Conference of the IEEE Engineering in Medicine \& Biology Society (EMBC). Montreal, QC, Canada: IEEE, 2020: 2113-6.

24Chen X, Xie C, Chen Z, Li Q. Automatic Tracking of Muscle Cross-Sectional Area Using Convolutional Neural Networks with Ultrasound. J Ultrasound Med 2019; 38: 2901-8.

25 Rohm M, Markmann M, Forsting J, Rehmann R, Froeling M, Schlaffke L. 3D Automated Segmentation of Lower Leg Muscles Using Machine Learning on a Heterogeneous Dataset. Diagnostics 2021; 11: 1747.

26Schindelin J, Arganda-Carreras I, Frise E, et al. Fiji: an open-source platform for biologicalimage analysis. Nat Methods 2012; 9: 676-82.

27 R Core Team. R: A language and environment for statistical computing. $R$ Foundation for Statistical Computing, Vienna, Austria 2019. URL https://www.R-project.org/.

28Bland MJ, Altman DG. Statistical methods for assessing agreement between two methods of clinical measurement. Lancet 1986; : 307-10.

29Hopkins WG, Marshall SW, Batterham AM, Hanin J. Progressive Statistics for Studies in Sports Medicine and Exercise Science. Med Sci Sports Exerc 2009; 41: 3-12.

3oZaidman CM, van Alfen N. Ultrasound in the Assessment of Myopathic Disorders: $J$ Clin Neurophysiol 2016; 33: 103-11. 